\title{
Comparing Mentalizing Abilities in Older Adults with and without Common Mental Disorders
}

\author{
Meinolf Peters ${ }^{\mathrm{a}}$ Holger Schul $z^{\mathrm{b}}$ \\ anstitut für Alterspsychotherapie und Angewandte Gerontologie, Marburg, Germany; ${ }^{b}$ Department of Medical \\ Psychology, Universitätsklinikum Hamburg-Eppendorf, Hamburg, Germany
}

\section{Keywords}

Mentalization - Theory of mind - Executive functions .

Depressive $\cdot$ Anxiety $\cdot$ Reflective functioning

\begin{abstract}
Introduction: The concept of mentalization is of central importance not only in modern psychotherapy but also in geriatric psychotherapy. In relation to older people, deficits in some aspects of the ability to mentalize, such as the theory of mind (ToM), affect perception, and metacognitive skills, are known. However, little is known about the mentalization ability of older people with mental disorders. The aim of the present study was to investigate the mentalizing abilities of older patients with depressive and anxious symptoms. Methods: In this study, the ability to mentalize using the Mentalization Questionnaire (MZQ), the HEALTH-49 questionnaire, Trail-Making Test (executive functions), and Reading-the-Mind-in-the-eyes-test (ToM) was studied in a clinical ( $n=150)$ and a nonclinical comparison sample of people ( $n$ $=150)$ in different age groups $(40-54,55-69, \geq 70)$. The evaluation of the data was conducted with different statistical methods, especially variance and regression analyses. $\boldsymbol{R} \boldsymbol{e}-$ sults: The clinical sample of all age groups showed significantly poorer mentalizing skills than the nonclinical samples. There were large correlations to health-related aspects. Ad-
\end{abstract}

Karger@karger.com www.karger.com/psp

Karger $\stackrel{\text { ' }}{5}$

GOPEN ACCESS
C 2022 The Author(s).

Published by S. Karger AG, Basel

This article is licensed under the Creative Commons Attribution 4.0 International License (CC BY) (http://www.karger.com/Services/ OpenAccessLicense). Usage, derivative works and distribution are permitted provided that proper credit is given to the author and the original publisher. ditionally, in the regression analysis, only the setting variable (clinical vs. nonclinical) had an influence on the MZQ score and neither gender, education, executive functions nor ToM had a significant influence. Age also had no effect and did not correlate with MZQ scores but did correlate negatively with ToM. Conclusions: The results show the importance of mentalization concepts for mental disorders in patients in the second half of life. There are indications of an imbalance of different aspects of mentalizing ability, especially between self- and other-related mentalizing abilities. Improving this balance could be a goal of therapeutic interventions.

(c) 2022 The Author(s).

Published by S. Karger AG, Basel

\section{Introduction}

The concept of mentalization has recently received increasing attention. According to Bateman and Fonagy [1], mentalization can be understood as the mental process by which an individual implicitly and explicitly ascribes meaning to his or her own and others' behaviours in relation to intentional states such as personal wishes, needs, feelings, beliefs, and other motivations [1]. Nevertheless, mentalization cannot be understood as a coherent construct. Rather, a number of "higher-order cognitions," such as the theory of mind (ToM), metacognition, 
self-reflection, mindfulness, or empathy, are connected with it. To date, it has not been clarified whether these different aspects are based on a general ability or whether they are a profile of abilities similar to personalities. Nevertheless, a number of distinctions can be made: automatic/implicit versus controlled/explicit mentalizing, internal versus external focus, self-versus other orientation, and cognitive versus affective mentalizing $[2,3]$. As a result of an empirical analysis of the construct, Lindeman and Lipsanen [4] distinguished 4 higher order constructs (cognitive and affective empathy, practical skills such as the understanding of metaphors, irony or humour, and metacognitions, especially self-reflection skills) and 14 lower order constructs, i.e., mentalization can be understood only as a multidimensional construct.

With regard to older people, the concept has thus far received little attention in its complexity; only individual aspects have been investigated to date. The concept of ToM has received special attention. ToM describes the ability to understand the mental states of others. In a meta-analysis, Henry et al. [5] concluded that such limitations are part of the normal ageing process. However, this provides only empirical evidence for one aspect of the mentalization concept, namely, the mentalization of others. However, in self-referential mentalization, there are results in only some aspects. Studies have shown deficits in metacognitive abilities [6] and empathy but only in the cognitive component of empathy $[7,8]$. There are also deficits in perception and speaking about feelings, especially negative feelings such as anger and sadness [9], but there is an improvement in the regulation of affect (RA), but only when there are moderate affects $[10,11]$.

In the meantime, there is a considerable amount of research literature on the ability to mentalize with regard to its significance in different clinical groups. In younger patients, there are numerous findings that show limitations in their ability to mentalize, e.g., in borderline patients or in depressive patients $[3,12]$. However, hardly any studies are available on elderly individuals. Using the Reading-the-Mind-in-the-Eyes Test (RMET), Peters and Schulz [13] were able to show that deficits in ToM skills were significantly more pronounced in a clinically stressed group of older people than in a clinically unstressed group. Cheavens et al. [14] showed that older people with Axis I disorders also have deficits in affect regulation, and Varkal et al. [15] confirmed that older patients with generalized anxiety disorders are less able to control their own thoughts due to negative metacognitive assumptions, which favours the development of anxiety. Overall, however, there are only a few studies in clin- ical groups of older people who applied the concept of mentalization.

The fact that empirical research on the ability to mentalize, especially in old age, is deficient may also have to do with the fact that it is a vague concept, according to Lindeman and Lipsanen [4], and it is difficult to grasp empirically. For a long time, only the Reflection Functioning scale [16] was available to measure mentalization ability, a complex rating procedure based on an attachment interview. Therefore, it seems very important that short self-evaluation procedures are now available, which allow for a more economic assessment of the construct [17]. A short instrument that has proven its worth is the Mentalization Questionnaire (MZQ) [18], which has been used repeatedly in younger clinical samples [19]. This instrument was used in the following study to assess for the first time the mentalization ability of patients in the second half of life with clinical symptoms compared to a nonclinical sample. Since the MZQ refers more to self-related mentalizing ability - including self-reflection and affective and pre-mentalizing aspects - the question of its relationship to other-related mentalizing abilities, i.e., ToM was of interest since the balance of both components can be understood as an expression of good mentalizing ability [1]. Since ToM abilities are closely related to executive functions [13], the question was whether these functions are also related to other mentalizing aspects. Finally, the question of whether correlations of sociodemographic variables, in particular age, with self-related mentalizing abilities could also be demonstrated was of interest; it was expected that the correlations would be negative. The following research questions were investigated:

1. Does a clinical versus a nonclinical group differ significantly on different mentalizing dimensions across different age groups?

2. Which sociodemographic characteristics, including age and health-related characteristics, are connected to mentalizing abilities?

3. Are mentalizing abilities associated with other constructs, including ToM and executive functions?

\section{Methods}

Design and Participants

A 2-factorial design was used to investigate the formulated questions. Factor 1 can be described as a setting factor, i.e., 1 clinical sample and 1 nonclinical sample were examined. The former consisted of a consecutive sample of patients from a psychosomatic clinic $(n=150)$. Thus, it is a heterogeneous sample with different 
Table 1. Comparison of sociodemographic characteristics of the clinical and nonclinical sample

\begin{tabular}{|c|c|c|c|}
\hline Baseline characteristics & Clinical sample, $n$ (\%) & Nonclinical sample, $n$ (\%) & $x^{2} / p / V$ \\
\hline Sample & $150(100)$ & $153(100)$ & \\
\hline \multicolumn{4}{|l|}{ Age groups } \\
\hline $40-54$ years & $50(33.3)$ & $49(32)$ & \multirow[t]{3}{*}{$0.531, p=0.767, V=0.042$} \\
\hline $55-69$ years & $50(33.3)$ & $57(37.3)$ & \\
\hline$\geq 70$ years & $50(33.3)$ & $47(30.7)$ & \\
\hline \multicolumn{4}{|l|}{ Sex } \\
\hline Men & $47(33.1)$ & $57(37.7)$ & \multirow[t]{2}{*}{$0.691, p=0.406, \mathrm{phi}=0.049$} \\
\hline Women & $95(66.9)$ & $94(62.3)$ & \\
\hline \multicolumn{4}{|l|}{ Education } \\
\hline Primary School & $71(50.4)$ & $48(31.8)$ & \multirow[t]{3}{*}{$13.105, p=0.001, V=0.212$} \\
\hline Middle School & 45 (31.9) & $53(35.1)$ & \\
\hline Higher School & $25(17.7)$ & $50(33.1)$ & \\
\hline \multicolumn{4}{|l|}{ Partnership } \\
\hline No partner & $12(8.5)$ & $10(6.6)$ & \multirow[t]{4}{*}{$5.390, p=0.145, V=0.136$} \\
\hline Married/partnership & $86(61.0)$ & $111(73.5)$ & \\
\hline Separated/divorced & $27(19.1)$ & $18(11.9)$ & \\
\hline Widowed & $16(11.3)$ & $12(7.9)$ & \\
\hline
\end{tabular}

clinical presentations of the ICD-10 chapters F30 and F40. This seems to be justified because in older patients, these clinical pictures are less defined but have more overlap. More than $80 \%$ of depressed older patients show anxiety symptoms at the same time [20]; the comorbidity of depression and anxiety disorders is significantly higher in older people than in younger people [21], and the involvement of somatic symptoms also increases with age.

For comparison, a nonclinical sample $(n=153)$ was enrolled, and they were recruited in different contexts (personal contacts, church choirs, meeting places, etc.). A prerequisite for inclusion was that no psychotherapeutic or psychiatric treatment had taken place in the last 3 years. In addition, the comparative sample was stratified in terms of sex and educational level. The patients were divided into 3 age groups: $40-54,55-69$, and 70 and older. There are indications [22] that the first losses of ToM skills can be observed as early as the middle of the fifth decade of life. In this respect, it can be assumed that the youngest age group does not have any age-related limitations but that the deficits then increase with age. This should make it possible to depict its development in the second half of life, albeit in a cross-sectional design. In both samples of all subjects over 65 years of age, the Mini-Mental State Examination [23] was used as a screening instrument to exclude participants with symptoms of dementia. Subjects with a value less than 27 were not included in the sample. No patients who had a personality disorder as their initial diagnosis were included in the clinical sample. The survey was conducted between the end of 2016 and the beginning of 2019 .

The 2 samples differed only slightly in terms of sex distribution, and low school-leaving qualifications were less common in the nonclinical sample than in the clinical sample. With regard to partnership status, the proportion of married or cohabiting partners was slightly higher in the nonclinical sample, but the proportion of those living apart or divorced was also higher (Table 1).
In the clinical sample, the diagnosis was also important. All patients come to the clinic with a preliminary diagnosis, which is reviewed and, if necessary, changed at the end of the treatment in a clinical consensus procedure based on ICD-10 criteria. The following diagnoses were predominant: $77 \%$ affective disorders (including 35\% with F31 and 40\% with F32), 7\% anxiety disorders, $5 \%$ adjustment disorders, and $5 \%$ somatoform disorders. A physical comorbidity affected $87 \%$, with migraine and headache being the most frequent (40\%).

\section{Instruments}

\section{Mentalization}

The MZQ was used, which was developed by Hausberg et al. [18] as an economical, feasible instrument, with a total of only 15 items. The questionnaire contains 4 analytically derived 4 -item scales with good reliability (internal consistency 0.60-0.68) and validity values. The scales are as follows: (1) refusing self-reflection (RSF) (4 items): the avoidance of thinking about one's own mental state (e.g., "Talking about feelings would mean that they become increasingly powerful"), (2) emotional awareness (EA) (4 items): the identification of feelings as well as a feeling of belonging is not present (e.g., "Sometimes it is only afterwards that I realize what feelings I had before"), (3) psychic equivalence (PA) mode (4 items): in mentalization theory, this occurs when one's own mental state is equated with external reality so that everything seems real (e.g., "I find it hard to believe that relationships can change"), and (4) RA (3 items): the inability to modulate one's own affects so that the person feels threatened by one's feelings (e.g., "Sometimes I cannot control my feelings well"). All questions are answered on a Likert scale of $1-5$, and the scale values must then be inverted so that a high score indicates a good ability to mentalize. The 4 scales can be combined to form a total score. 
Table 2. Means and standard deviations of the psychometric scales in the clinical and nonclinical sample

\begin{tabular}{llllll}
\hline \multirow{2}{*}{ Scale } & \multicolumn{2}{c}{ Clinical sample } & & \multicolumn{2}{c}{ Nonclinical sample } \\
\cline { 2 - 3 } \cline { 6 - 6 } & M & SD & & $M$ & SD \\
\hline RMET & 18.93 & 4.08 & & 20.64 & 4.73 \\
TMT & 132.43 & 84.02 & & 111.20 & 65.85 \\
HEALTH-PHO & 1.00 & 1.01 & & 0.14 & 0.35 \\
HEALTH-DEP & 1.94 & 1.98 & & 0.34 & 0.45 \\
HEALTH-SOM & 1.80 & 0.95 & & 0.88 & 0.65 \\
HEALTH-WB & 1.30 & 0.82 & & 2.75 & 0.64 \\
HEALTH-IP & 1.95 & 1.02 & & 0.72 & 0.65 \\
HEALTH-A\&P & 2.22 & 0.86 & & 0.91 & 0.69 \\
\hline
\end{tabular}

$\mathrm{H}-\mathrm{PHO}$, phobic anxiety; H-DEP, depressive symptoms; H-SOM, somatic complaints; $\mathrm{H}-\mathrm{WB}$, well-being; $\mathrm{H}-\mathrm{IP}$, interpersonal problems; $\mathrm{H}-\mathrm{A} \& \mathrm{P}$, social activities and participation.

Theory of Mind

The RMET was used. It was developed by Baron-Cohen et al. [24] as part of autism research but has since been used in numerous other contexts and translated into German and tested by Bölte [25]. Thirty-six photos show the eye areas of faces expressing different emotional states. Four terms are available, 1 of which is to be assigned to the facial expression. A total score (maximum 36 points of correct answers) was calculated, which was used here. The results of the reliability and validity tests are satisfactory [26], and it has also been used in older subjects [5].

Psychosocial Health

HEALTH-49 is a questionnaire for recording psychosocial health [27] that comprises 6 scales: HEALTH-PHO (phobic anxiety), HEALTH-DEP (depressive symptoms), HEALTH-SOM (somatic symptoms), HEALTH-WB (well-being), HEALTH-IP (interpersonal problems), and HEALTH-A\&P (activity and participation). The internal consistency of the scales was predominantly high, with values between 0.76 and 0.91 .

\section{Executive Functions}

The Trail-Making Test (TMT) [28] is an economical instrument to measure some aspects of executive functions, especially divided attention and cognitive flexibility. It involves connecting randomly arranged numbers and letters in the shortest possible time and in the correct order, always alternating between numbers and letters. In the analysis, we used only time as a measure.

\section{Statistical Analysis}

The analyses were carried out using SPSS (Version 25). Twofactor analyses of variance were calculated for the total value of the MZQ and the 4 scales of this questionnaire. For post hoc comparisons of the 3-step age factor, the LSD was selected. To analyse multivariate relationships, linear regression analyses (model: enter) were calculated using the MZQ-total scale as the criterion and age, sex, education, TMT, and RMET as predictor variables. The 3 -level variable education was converted into 2 dummy variables.
For this purpose, the assumed years of schooling associated with the different degrees achieved were used (primary school $=9$, middle school $=10$, and higher school $=12$ years). The chosen sample size allows for the detection of differences of the mean with a medium effect size at an alpha level of $5 \%$ and a power of $80 \%$, both in the analyses of variance and the $t$ test in the chosen primary outcomes. In the linear regression models, the sample size enables the detection of a small effect size of $f=0.05$ (with alpha $5 \%$, power $80 \%$, and 10 predictor variables).

\section{Results}

\section{Descriptive Values of the Scales}

Table 2 shows the descriptive values (means and standard deviations) of the survey instruments used (RMET, TMT, HEALTH scales).

\section{Differences in Mentalization in Age and Settings}

First, the setting and age differences were checked using two-factor variance analyses. When comparing the clinical and nonclinical samples, the results were highly significant (see Table 3), with lower values in the clinical group based on the total value of the MZQ $(F(1,292)=$ $\left.80.95, p<0.000, \mathrm{pEta}^{2} 0.221\right)$, as well as the scales refusing self-reflection $(\mathrm{RSF})\left(F(2,292)=39.35, p<0.000, \mathrm{pEta}^{2}\right.$ $0.121)$, EA $\left(F(2,292)=73.17, p<0.000, \mathrm{pEta}^{2} 0.204\right)$, emotional awarness (EA) $(F(2,292)=73.17, p<0.000$, pEta $\left.^{2} 0.204\right)$, psychic aquivalence mode $(\mathrm{PA})(F(2,292)=$ 57.35, $\left.p<0.000, \mathrm{pEta}^{2} 0.167\right)$, and regulation of affect (RA) $\left(F(2,292)=59.24, p<0.000, \mathrm{pEta}^{2} 0.171\right)$. The situation is different for age differences, which were not significantly differences either in the total value or in the individual scales. The MZQ overall value $(F(2,292)=$ $0.251, p=0.59)$ shows just as few age differences for the RSF (self-reflection) scale $(F(2,292)=2.40, p=0.092)$, although a tendency becomes apparent here; the oldest group shows the lowest values compared to the other 2 groups ( $p=0.046$ between the youngest and oldest and $p$ $=0.039$ between the middle and oldest groups). For the EA scale $(F(2,292)=0.26, p=0.771)$, there was no age difference. In the scale PA mode (reflexive mode) ( $F(2$, $292)=2.47, p=0.086$ ), there was a tendency, but in this case, in favour of the oldest group, which differed from the youngest group in a post hoc comparison with $p=$ 0.015 . The RA scale $(F(2,292)=1.36, p=0.258)$ again does not show a significant age difference. Exploratory tests were also carried out to determine whether there was an interaction effect between the setting and age, which was not the case, either in the overall value or in the individual scales. 
Table 3. Means and standard deviations of the MZQ-total score and the MZQ subscales for the different age groups in the clinical and nonclinical sample

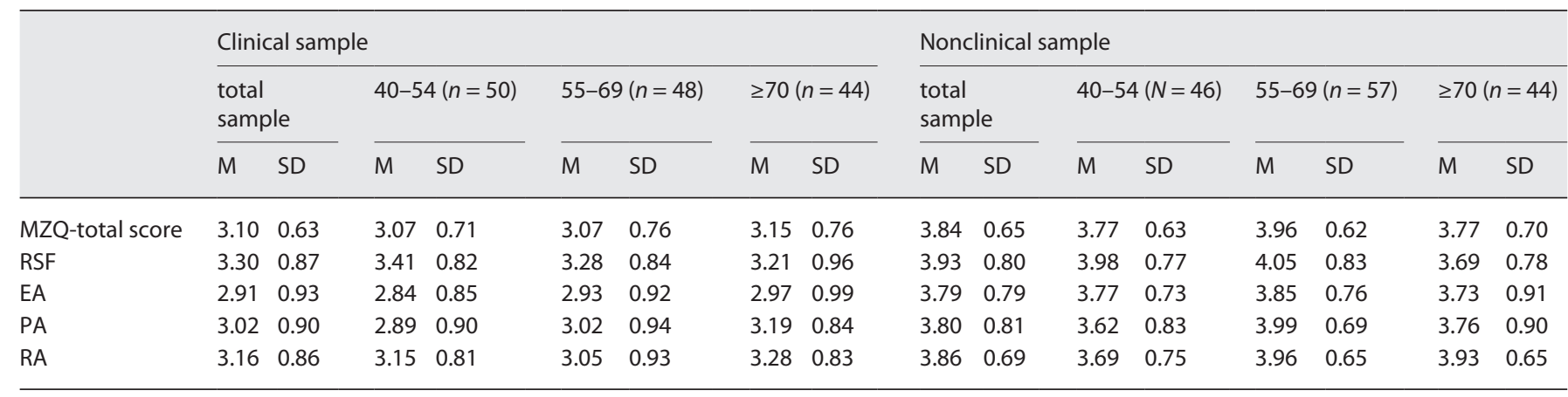

RSF, refusing self-reflection; EA, emotional awareness; PA, psychic equivalence; RA, regulation of affect.

Table 4. Intercorrelations of sociodemographic and clinical variables in the total sample

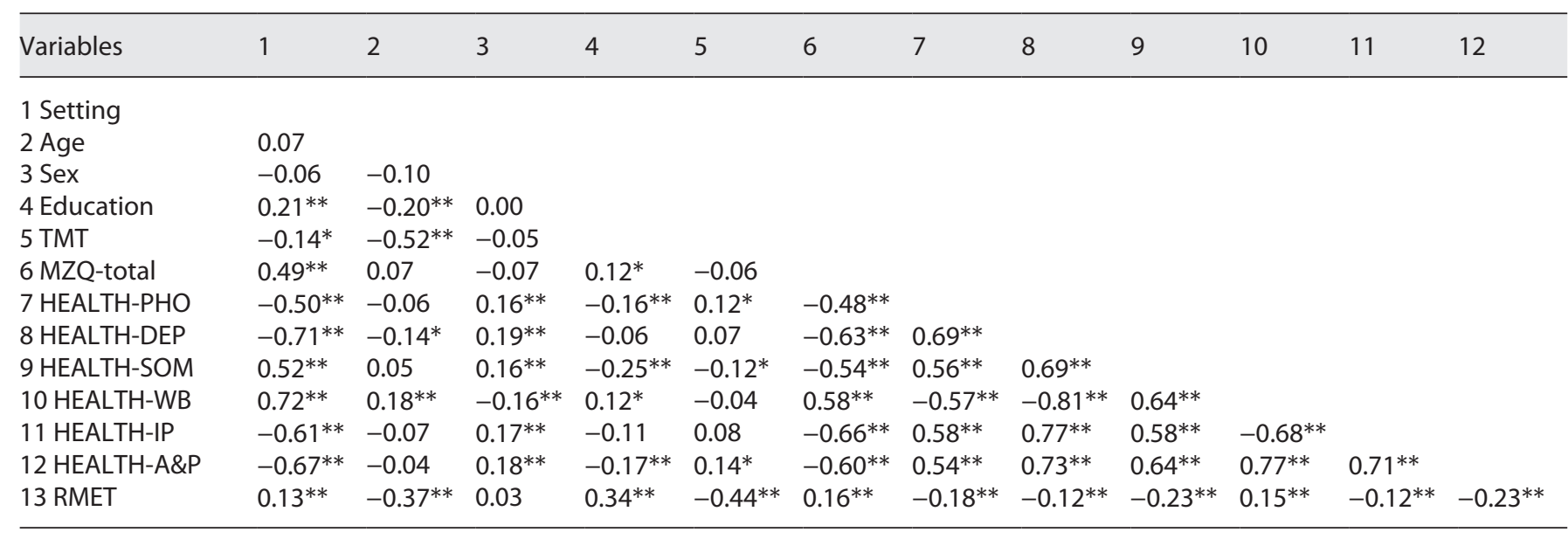

Setting, 1 = clinical, 2 = nonclinical; Sex, men = 1, women = 2; H-PHO, phobic anxiety; H-DEP, depressive symptoms; H-SOM, somatic complaints; H-WB, well-being; H-IP, interpersonal problems; H-A\&P, activities and participation. Significance: ${ }^{*} p<0.05, * * p<0.01$.

Mentalization and Person-, Symptom- and Health-

Related Variables

Finally, the connections between the ability to mentalize and person-, symptom- and health-related variables were investigated. Table 4 shows the intercorrelations of the included variables.

Looking at the correlations of the MZQ-total with the other variables, the consistently significant correlations with large effects fall within the HEALTH scales; on the symptom-related scales, there are negative correlations with medium or effects. The strongest correlation is with the variable HEALTH-DEP, i.e., subjects with good mentalization skills show a lower symptom burden. Similarly, there are strong correlations with high effect strength with the HEALTH scales well-being, interpersonal problems and activity \& participation, i.e., subjects with good mentalization skills show a better sense of well-being; they have fewer interpersonal problems and are socially more integrated. All of these variables have a very low correlation with age, except HEALTH-DEP, which correlates negatively, and HEALTH-WB, which correlates significantly positively with age, but both with a small effect size.

The relationship between the MZQ and the RMET was $0.16^{*}$, which was significant at the $5 \%$ level. Self-referral mentalization ability, measured with the MZQ, and ToM abilities correlated moderately but significantly with each other. With respect to the subscores of the MZQ, this correlation was confirmed only for the RSF scale. The correlation of $0.24^{* *}$ was significant but also with a small effect size. The other 3 scales correlated only at 0.10 and 0.11 with the overall RMET value. Table 5 shows the correla- 
Table 5. Correlations of the MZQ subscales with sociodemographic and clinical variables

\begin{tabular}{lllll}
\hline Variable & RSF & EA & PA & RA \\
\hline 1 Setting & $0.35^{* *}$ & $0.46^{* *}$ & $0.41^{* *}$ & $0.41^{* *}$ \\
2 Age & -0.09 & 0.05 & 0.11 & $0.13^{*}$ \\
3 Sex & 0.01 & -0.08 & -0.10 & -0.02 \\
4 TMT & $-0.16^{*}$ & -0.01 & -0.05 & 0.02 \\
5 RMET & $0.24^{* *}$ & 0.10 & 0.10 & 0.11 \\
6 HEALTH-PHO & $-0.40^{* *}$ & $-0.39^{* *}$ & $-0.46^{* *}$ & $-0.38^{* *}$ \\
7 HEALTH-DEP & $-0.43^{* *}$ & $-0.58^{* *}$ & $-0.60^{* *}$ & $-0.55^{* *}$ \\
8 HEALTH-SOM & $-0.43^{* *}$ & $-0.51^{* *}$ & $-0.46^{* *}$ & $-0.45^{* *}$ \\
9 HEALTH-WB & $0.41^{* *}$ & $0.53^{* *}$ & $0.52^{* *}$ & $0.48^{* *}$ \\
10 HEALTH-IP & $-0.49^{* *}$ & $-0.61^{* *}$ & $-0.62^{* *}$ & $-0.60^{* *}$ \\
11 HEALTH-A\&P & $-0.47^{* *}$ & $-0.51^{* *}$ & $-0.54^{* *}$ & $-0.52^{* *}$ \\
\hline
\end{tabular}

RSF, refusing self-reflection; EA, emotional awareness; $P A$, psychic equivalence; RA, regulation of affect. Setting, $1=$ clinical, 2 = nonclinical; Sex, men $=1$, women $=2 ; \mathrm{H}-\mathrm{PHO}$, phobic anxiety; $\mathrm{H}$-DEP, depressive symptoms; H-SOM, somatic complaints; H-WB, well-being; H-IP, interpersonal problems; H-A\&P, activities and participation. Significance: ${ }^{*} p<0.05,{ }^{* *} p<0.01$.

tions of the MZQ scales with the other variables included. All scales correlate highly significantly and with medium effect sizes with the setting variable, i.e., mentalizing ability is lower in the clinical sample than in the nonclinical sample. They also correlate highly significantly with the HEALTH scales, partly with large effect sizes.

There are still significant correlations with medium effect size with the variable setting, i.e., in the nonclinical sample, the MZQ values were more pronounced. Finally, there are highly significant correlations, some with large effect sizes, with the scales of the HEALTH-49, i.e., mentalization skills are closely related to health-related aspects.

In the next step, a multiple regression analysis was performed (Table 6). As the criterion MZQ-total was used as a predictor, the variables already listed in the regression analysis were introduced. However, since the variable setting (clinical and nonclinical) correlated strongly with the HEALTH scales, these variables were omitted due to the problem of multicollinearity. Since the relationship to ToM abilities was of particular interest, as well as the question of whether this is age-dependent, the interaction variable Age-X-RMET was additionally introduced as a predictor. The variable setting alone had a significant beta weight, i.e., the subjects of the nonclinical sample had higher MZQ values than those of the clinical sample. Neither RMET nor the Age-X-RMET interaction variable had a significant beta weight.
Table 6. Regression coefficients of sociodemographic and clinical variables on MZQ-total

\begin{tabular}{lllll}
\hline Predictor & $B$ & SD & $\beta$ & Cl 95\% \\
\hline 1 Setting & 0.718 & 0.088 & $0.453^{* *}$ & $0.546,0.891$ \\
2 Age & -0.019 & 0.019 & -0.275 & $-0.056,0.018$ \\
3 Sex & -0.046 & 0.089 & -0.028 & $-0.222,0.130$ \\
4 Education middle & 0.004 & 0.103 & 0.002 & $-0.200,0.207$ \\
5 Education high & 0.017 & 0.115 & 0.010 & $-0.209,0.244$ \\
6 TMT & 0.000 & 0.001 & 0.027 & $-0.001,0.002$ \\
7 RMET & -0.053 & 0.054 & -0.297 & $-0.160,0.054$ \\
8 Age $\times$ RMET & 0.001 & 0.001 & 0.428 & $-0.001,0.003$ \\
\hline
\end{tabular}

Setting, 1 = clinical, 2 = nonclinical; Sex, men $=1$, women $=2$; Education, middle $=10$, high $=12$ years. $R=0.49, R^{2}=0.24, F(8,271)$ $=10.768^{* * *}$. Significance: ${ }^{* *} p<0.01,{ }^{* * *} p<0.001$.

\section{Discussion}

The concept of mentalization ability was developed from psychoanalysis and is, today, one of the central concepts in modern psychotherapy. It is assumed to be of particular importance for the development of mental disorders and thus also for their treatment. There are also voices from behavioural therapy who see, in this construct, a common basis for the future development of psychotherapy [29]. To date, however, there are hardly any findings on how the ability to mentalize changes in old age and what significance it has in connection with clinical disorders. The present study should serve to fill in some of these knowledge gaps.

In the first hypothesis, it was tested whether the 2 samples differed from each other, which was confirmed both for the total value of the MZQ and for the 4 subscores. Clinically impaired participants in the second half of life consistently had lower scores in various aspects of mentalization abilities than clinically unimpaired participants. This confirms that in this phase of life, the ability to mentalize may be considered a resilience factor with regard to the development of psychological symptoms [30]. The correlations of the MZQ with the scales of the HEALTH-49 are all highly significant with large effect sizes. Good mentalizing abilities are associated with low symptom burden, high well-being, fewer interpersonal problems, and greater levels of social activity and participation. However, due to the cross-sectional study design, no statement can be made about causality. Thus, it cannot be assumed that such deficits alone will impair the ability to deal with emotions and social conflicts and thus promote the development of mental symptoms. It is also con- 
ceivable that this has an effect on the ability to mentalize and thus, for example, limits the absorption of social information or the perception of certain affects. The question of causality could be clarified only in a longitudinal study design.

The importance of the ability to mentalize in terms of psychological symptoms is also evident from its correlations with the scales of HEALTH-49, which are all in the range of strong effects. The highest correlation is with the HEALTH-IP scale, i.e., with interactional personal problems that show a particularly close connection to the ability to mentalize. With regard to the symptom scales, there is the strongest correlation with the HEALTH-DEP scale, i.e., depressive symptomatology. This also confirms the findings obtained in younger patients, among whom depressive and anxious patients demonstrated limited mentalization abilities $[3,12]$. With regard to depressive disorders, Luyten et al. [31] developed a theory that attributes reduced mentalization abilities in this disorder to early attachment experiences or breaks in relationships, which results in the selffeeling threatened, especially in stressful situations, and the ability to mentalize being limited. Stressful situations, however, have been experienced by all patients in the clinical sample and are an important background to inpatient treatment. In the case of older patients, these are often loss events such as the death of the partner or stress due to caregiving tasks, for example. Such stressful events, as well as potentially traumatic events earlier or in mid-life [32], can impair the ability to mentalize in older age.

A further focus of the study was to study age changes. However, the age changes were only slightly pronounced. The symptom-related scales were only moderately correlated with age; depressive symptoms decreased with increasing age, while subjective well-being increases and is positively correlated with age, but only with a small effect. Above all, the latter agrees with gerontological findings on the development of well-being in old age [33]. However, there were no age differences in mentalization abilities. How can the absence of an age effect be explained? There is a striking contrast in the findings on ToM abilities, which almost all showed deficits in old age [5]. The correlation of the RMET and MZQ subscales is significant only on the RSF scale. This scale also includes the teleological aspect of being able to distinguish between the behaviour and intentions of the other persons, i.e., it is also about other-related mentalizing. This means that there is also a conceptual overlap with ToM. In the regression analysis, neither the RMET variable nor the Age-XRMET interaction variable showed a significant connection with MZQ.

Mentalization Abilities in Older Patients
The question is, why are self- and other-related mentalizing not more closely related? In addition to differences in the operationalization of both aspects (see below), neuropsychological age changes might be of importance. An explanation could be that inhibition skills, which decline with growing age, are responsible for ToM deficits in old age [34] because the assessment of the mental state of the counterpart depends on the inhibition of self-referential cognitions [35]. This explanation, however, also includes the statement that self-referral cognitions are not restricted by that deficit, i.e., the self-perceived ability to mentalize is not subjected to age restrictions either. This consideration is supported by the finding that the MZQ does not correlate with the TMT, i.e., the self-perceived ability to mentalize is not related to executive functions, whereas TMT performance correlates highly with ToM performance, indicating that the self-related ability to mentalize is subject to fewer limitations in executive functions. This could also have a positive effect if, for example, it is perhaps associated with an increase in introversion, wisdom, or life review, which are all based on the crystallized intelligence developed in old age, whereas ToM performance tends to presuppose fluid abilities that are deficient in old age. On the other hand, an inner imbalance could arise because self-centred mentalizing is less coordinated with or regulated by external mentalizing, i.e., in old age, the integration of self-centred and other-centred mentalizing could be reduced or an imbalance of mentalizing with external and internal focus could arise. Such partial disintegration could, however, have negative social consequences, as found by von Hippel and Dunlop [36]. They were able to show that in social situations, older people occasionally tend to make thoughtless statements, such as expressing stereotypical ideas that are more likely to be inhibited by younger people. This could also be associated with a more egocentric way of speaking, as reported in studies on communication in old age [37], or an increase in verbose speech [38]. Such problematic behaviours could well have negative consequences in social life, as other studies have shown that deficits in social cognition are connected with reduced participation in social activities [39]. However, this interpretation remains speculative and should be the subject of future studies.

The study is subject to some limitations. Firstly, this was a cross-sectional study; therefore, the differences in the age groups could not be interpreted as developmental differences. Furthermore, it is not ensured that the comparative sample was representative. Another problem is the operationalization of the variables. Although the va- 
lidity of the MZQ is generally proven [18], the question arises whether this applies to older people in the same way. It should be borne in mind that older people often have a "positive bias," which may lead to a self-protective overestimation of their own abilities ("positivity effect"), which may also be a reason for the lack of age differences. To further examine age differences, other operationalizations would have to be carried out. ToM skills, on the other hand, were captured via a direct behavioural measure, i.e., in a different way than self-centred mentalizing. This different recording of both aspects of mentalizing ability might have contributed to the fact that the correlation is only slightly pronounced.

Despite these limitations, the results provide a first insight into the mentalization ability of older people and its significance for addressing clinical problems. The consideration of the ability to mentalize in geriatric psychotherapy should therefore be given high priority. The first goal would be to improve individual components of mentalizing abilities such as ToM skills to promote health. However, the second goal is to bring the different components into better balance. The better linking of self- and other-related mentalization skills, for example, could be a prerequisite for alleviating interaction problems, improving social integration and thus also reducing depressive and anxious symptoms. That this is possible was shown in the "Hersfelder Katamnesestudie," in which - also measured with the MZQ - the over-60 participants showed just as good effect sizes as younger patients after an inpatient treatment [40].

\section{Acknowledgments}

We would like to thank all the patients who participated in the study for their readiness and engagement as well as their patience and effort. In addition, we would like to thank the staff of the hospital in Bad Hersfeld (Hessen/Germany), who facilitated the assessment and supported data collection.

\section{Statement of Ethics}

The study was approved by the Independent Ethics Committee of Philipps-University Marburg/Germany (number AZ1-10-15). All participants completed an informed consent form that included the purpose and circumstances of the study, information regarding data privacy and the right to refuse participation without any consequences, as well as information regarding the right to withdraw from the study during and after data collection.

\section{Conflict of Interest Statement}

The authors state that there were no conflicts of interest.

\section{Funding Sources}

This study was supported by Heidehof-Stiftung, Stuttgart/Germany.

\section{Author Contributions}

M.P. and H.S. participated in the conception and planning of the study. M.P. performed the acquisition and M.P. and H.S. analysis of the data. M.P. and H.S. wrote the draft manuscript, which was revised and supplemented by both authors. Both authors approved the final manuscript and stand by the findings and conclusions. The authors alone are responsible for the content and writing of the report.

\section{Data Availability Statement}

The data that support the findings of this study are available from the corresponding author upon reasonable request.

\section{References}

1 Bateman AW, Fonagy P. Psychotherapie der borderline-persönlichkeitsstörung. Giessen: Psychosozial-Verlag; 2008.

2 Choi-Kain LW, Gunderson JG. Mentalization: ontogeny, assessment, and application in the treatment of borderline personality disorder. Am J Psychiatry. 2008;165:1127-35.

3 Fischer-Kern M, Tmej A. Mentalization and depression: theoretical concepts, treatment approaches and empirical studies: an overview. Z Psychosom Med Psychother. 2019;65: $162-77$.
4 Lindeman M, Lipsanen J. Mentalizing: seeking the underlying dimensions. Int J Psychol Stud. 2017;9(1):10-23.

5 Henry JD, Phillips LH, Ruffman T, Bailey PE. A meta-analytic review of age differences in theory of mind. Psychol Aging. 2013;28(3): 826-39.

6 Palmer EC, David AS, Fleming SM. Effects of age on metacognitive efficiency. Conscious Cogn. 2014;28:151-60.
7 Hühnel I, Fölster M, Werheid K, Hess U. Empathic reactions of younger and older adults: no age related decline in affective responding. J Exp Soc Psychol. 2014;50:136-43.

8 Khanjani Z, Jeddi EM, Hekmati I, Khalilzade S, Nia ME. Comparison of cognitive empathy, emotional empathy and social functioning in different age groups. Aust Psychol. 2014;50:80-5.

9 Kunzmann U, Rohr M, Wieck C, Kappes C, Wrosch C. Speaking about feelings: further evidence for multidirectional age differences in anger and sadness. Psychol Aging. 2017; 32(1):93-103. 
10 Gross JJ, Carstensen LL, Pasupathi M, Tsai J, Skorpen CG, Hsu AY. Emotion and aging: experience, expression, and control. Psychol Aging. 1997;12:590-9.

11 Charles ST. Strength and vulnerability integration: a model of emotional well-being across adulthood. Psychol Bull. 2010;136(6): 1068-91.

12 Staun L, Kessler H, Buchheim A, Kächele H, Taubner S. Mentalisierung und chronische depression. Psychotherapeut. 2010;55(4): 299-305.

13 Peters M, Schulz H. Theory-of-Mind abilities in elderly patients with common mental disorders: a cross sectional study. Aging Ment Health. 2021.

14 Cheavens JS, Rosenthal MZ, Banawan SF, Lynch TR. Differences in emotional experience and emotion regulation as a function of age and psychiatric condition. Aging Ment Health. 2008;12(4):478-87.

15 Varkal MD, Yalvac D, Tufan F, Turan S, Cengiz M, Emul M. Metacognitive differences between elderly and adult outpatients with generalized anxiety disorder. Eur Geriatr Med. 2013;4(3):150-3.

16 Katznelson H. Reflective functioning: a review. Clin Psychol Rev. 2014;34(2):107-17.

17 Gagliardini G, Colli A. Assessing mentalization: development and preliminary validation of the modes of mentalization scale. Psychoanal Psychol. 2019;36(3):249-58.

18 Hausberg MC, Schulz H, Piegler T, Happach CG, Klöpper M, Brütt AL, et al. Is a self-rated instrument appropiate to assess mentalization in patients with mental disorders? Development and first validation of the mentalization questionaire (MZQ). Psychother Res. 2012;22(6):699-709.

19 Hayden MC, Müllauer PK, Gaugeler R, Senft $B$, Andreas S. Mentalization as mediator between adult attachment and interpersonal distress. Psychopathology. 2019;52:10-7.
20 Braam AW, Copeland J, Delespaul P, Beekman A, Como A, Dewey M, et al. Depression, subthreshold depression and comorbid anxiety symptoms in older Europeans: results from the EURODEP concerted action. J Aff Disord. 2014;155:266-72.

21 Beekman ATF, Bremmer MA, Dorly JH. Anxiety disorders in later life: a report from the longitudinal aging study Amsterdam. Int J Geriatr Psychiat. 1998;13:717-26.

22 Pardini M, Nichelli PF. Age-related decline in mentalizing skills across adult life span. Exp Aging Res. 2009;35:98-106.

23 Folstein MF, Folstein SE, McHugh PR. Minimental-state: a practical method for grading the cognitive state of patients for the clinician. J Psychiatr Res. 1975;12(3):189-98.

24 Baron-Cohen S, Wheelwrigth J, Hill J, Raste $Y$. The "reading the mind in the eyes" test revised version: a study with normal adults, and adults with asperger syndrome or high-functioning autism. J Child Psychol Psychiatr Allied Disc. 2001;42(2):241-51.

25 Bölte S. Reading mind in the eyes test. Erwachsenenversion [online]. 2005 [cited 2013 Aug 10]. Available from: http://www.as-tt.de/ assets/applets/Augentest_Erwachsene.pdf.

26 Vellante M, Baron-Cohen S, Melis M, Marrone $\mathrm{M}$, Petretto DR, Masala $\mathrm{C}$, et al. The "reading the mind in the eyes" test: systematic review of psychometric study in Italy. Cog Neuropsychiatr. 2013;18(4):326-54.

27 Rabung S, Harfst T, Kawski S, Koch U, Wittchen H-U, Schulz H. Psychometrische überprüfung einer verkürzten version der "hamburger module zur erfassung allgemeiner aspekte psychosozialer gesundheit für die therapeutische praxis« (HEALTH-49). Zeit Psychosom Med Psychother. 2009;55(2): $162-79$.
28 Reitan RM. Trail making test (TMT). Göttingen: Hogrefe; 1979.

29 Wendisch M . Verhaltenstherapie emotionaler schlüsselerfahrungen. Vom kognitiven training zur emotionalen transformation. Bern: Huber; 2015.

30 Bateman AW, Fonagy P, editors. Handbook of mentalization in mental health. New York: American Psychiatric Publishing; 2011.

31 Luyten P, Lemma A, Target M. Mentalizing and depression. In: Bateman A, Fonagy P, editors. Mentalizing in mental health practice. Washington, DC: American Psychiatric Publishing Inc.; 2012. p. 385-418.

32 Peters M. Trauma and mentalization ability in older patients. An empirical contribution to the effect of trauma in old age. GeroPsych. 2021;34(4):189-99.

33 Kunzmann U, Little TD, Smith J. Is age-related stability of subjective well-being a paradox? Cross-sectional and longitudinal evidence from the Berlin aging study. Psychol Aging. 2000;15(3):511-26.

34 Hasher L, Beth Quing M, May C. Inhibitory control over no-longer-relevant information. Adult age differences. Memory Cog. 1997;25: 286-95.

35 Firth CD, Firth U. The neural basis of mentalizing. Neuron. 2006;50:531-4.

36 von Hippel W, Dunlop SM. Aging, inhibition, and social inappropriateness. Psychol Aging. 2005;20(3):519-23.

37 Thimm C. Alter - Sprache - Geschlecht. Frankfurt: Campus; 2000

38 Ruffman T, Murray J, Halberstadt J, Taumoepeau M. Verbosity and emotion recognition in older adults. Psychol Aging. 2010;25(2): 492-7.

39 Bailey PE, Henry JD, Von Hippel W. Empathy and social functioning in late adulthood. Aging Ment Health. 2008;12:499-503.

40 Peters M, Budde A, Lindner J, Schulz $\mathrm{H}$. Ergebnisse der hersfelder katamnesestudie [Preprint]. 2022. 